\title{
A Polar Codes-Based Distributed UEP Scheme for the Internet of Things
}

\author{
Chen Cui $\mathbb{D}^{1},{ }^{1}$ Shuang Wu $\mathbb{D}^{2}{ }^{2}$ Zhenyong Wang $\mathbb{D}^{1},{ }^{1}$ Qing Guo, ${ }^{1}$ and Wei Xiang $\mathbb{D}^{3,4}$ \\ ${ }^{1}$ School of Electronics and Information Engineering, Harbin Institute of Technology, Harbin 150001, China \\ ${ }^{2}$ College of Engineering, Xi'an International University, Xi'an 710070, China \\ ${ }^{3}$ School of Engineering and Mathematical Sciences, La Trobe University, Melbourne, VIC 3086, Australia \\ ${ }^{4}$ College of Science and Engineering, James Cook University, Cairns, QLD 4878, Australia \\ Correspondence should be addressed to Zhenyong Wang; zywang@hit.edu.cn
}

Received 19 May 2021; Accepted 29 July 2021; Published 16 December 2021

Academic Editor: Laurie Cuthbert

Copyright (C) 2021 Chen Cui et al. This is an open access article distributed under the Creative Commons Attribution License, which permits unrestricted use, distribution, and reproduction in any medium, provided the original work is properly cited.

\begin{abstract}
The Internet of Things (IoT), which is expected to support a massive number of devices, is a promising communication scenario. Usually, the data of different devices has different reliability requirements. Channel codes with the unequal error protection (UEP) property are rather appealing for such applications. Due to the power-constrained characteristic of the IoT services, most of the data has short packets; therefore, channel codes are of short lengths. Consequently, how to transmit such nonuniform data from multisources efficiently and reliably becomes an issue be solved urgently. To address this issue, in this paper, a distributed coding scheme based on polar codes which can provide UEP property is proposed. The distributed polar codes are realized by the groundbreaking combination method of noisy coded bits. With the proposed coding scheme, the various data from multisources can be recovered with a single common decoder. Various reliability can be achieved; thus, UEP is provided. Finally, the simulation results show that the proposed coding scheme is viable.
\end{abstract}

\section{Introduction}

In recent years, polar codes have attracted much attention, since they are the first kind of provably capacityapproaching error-correction codes of any given binaryinput discrete memoryless channels (B-DMCs) [1]. Moreover, polar codes can provide good error-correction performances under short code length scenarios, which makes polar codes suited for the Internet of Things (IoT) applications [2]. There are a massive number of devices in the IoT networks. Most of the data transmitted over the IoT networks are with short-packets. How to transmit these data with high efficiency and reliability becomes an issue worth studying [3].

Recently, as a large number of devices need to be connected with the network and transmit the data of these devices, the IoT paradigm has the opportunity to become reality [4]. The large number of IoT devices will bring about a series of problems, which have attracted much attention from the researchers. One is how to support a massive number of access devices with limited spectrum resources. The nonorthogonal multiple access (NOMA) technology has the potential to dramatically increase the amount of accessed IoT devices [5-7]. In addition, the increase in the number of devices in the Internet of Things will also lead to high costs, which cannot be ignored in the implementation of the Internet of Things [8]. In order to ensure low costs, the energy consumption and computing power of devices in the Internet of Things are severely limited $[9,10]$. Moreover, the growing number of IoT devices also leads to different requirements for data reliability among different devices. If the data can be transmitted according to their own requirements, the limited resources can be effectively utilized. Unequal error protection (UEP) is an efficient way to transmit data in the IoT scenarios.

In recent decades, different UEP coding schemes have been proposed on Turbo codes [11], LDPC codes [12, 13], rateless codes [14, 15], and polar codes [16]. All the above- 
mentioned codes which can provide UEP property are centralized codes. However, in the IoT scenarios, different devices need to transmit data to a common destination, where distributed coding schemes are needed. Assume there are multi-independently and identically distributed sources. The data of each source are encoded independently. If the coded data can be decoded with a single decoder employing the correlations between the sources, then this coding scheme can be considered as a distributed code [17].

The massive number of devices in the IoT have low costs demands. Distributed coding can reduce the number of decoders and control the number of access devices flexibly. Distributed codes have attracted much attention in the last decade, especially for the video data transmission systems $[18,19]$. Distributed codes with UEP property have also been proposed. Talari proposed the first distributed UEP rateless codes over the two-source and single-relay scenario by assigning different selecting probabilities in the recoded process on the relay node. In this way, the data from different sources can be decoded with different reliability levels [20, 21]. As the unequal selecting process on the relay node may dramatically affect the overall transmission efficiency of the distributed code. In [22], a kind of distributed UEP rateless codes in which all the subcodes are with the equal error protection (EEP) property are proposed. The drawbacks of unequal selecting process can be avoided by careful design of the code parameters.

In this paper, a polar-based distributed coding scheme with UEP property is proposed. Unlike the rateless codes which are designed to settle packet erasure issues, the distributed polar codes are designed to prevent bit errors. A very important difference exists between the proposed distributed UEP codes and other existing UEP coding schemes, which is the UEP property of proposed codes is provided by the inner disparities of information bits and does not need to change the coding structures of polar codes, while the UEP properties of the other existing UEP codes are obtained by redesigning the coding structures or parameters.

The organization of this paper is summarized as follows. In Section 2, we briefly review the fundamentals of polar codes. Then, the proposed distributed coding scheme is introduced in Section 3. Some necessary definitions and settings have been provided. A groundbreaking method of combining the collected noisy bits is put forward. In Section 4, there are some related discussions about the proposed scheme. Section 5 investigates the simulation results. And the paper concludes in Section 6.

\section{Preliminaries}

In this section, we briefly review the fundamentals of polar codes, including the following aspects: channel polarization, construction, and decoding of polar codes.

2.1. Channel Polarization. Channel polarization is realized by operations of channel combination and channel splitting [1]. Let $(\mathscr{X}, \mathscr{Y}, W(y \mid x))$ denote a B-DMC channel, where $\mathscr{X}$ and $\mathcal{Y}$ are the input and output alphabets and $W(y \mid x)$ is the transition probabilities. Let $W^{N}$ denote $N$-independent use of channel $W$, we have

$$
W^{N}\left(y_{1}^{N} \mid x_{1}^{N}\right)=\prod_{i=1}^{N} W\left(y_{i} \mid x_{i}\right)
$$

where $x_{1}^{N}=\left(x_{1}, \ldots, x_{N}\right)$ and $y_{1}^{N}=\left(y_{1}, \ldots, y_{N}\right)$. These $N$ independent channels can be combined into a single synthesized channel $W_{N}$, which can be represented as

$$
W_{N}\left(y_{1}^{N} \mid u_{1}^{N}\right)=W^{N}\left(y_{1}^{N} \mid u_{1}^{N} G_{N}\right)
$$

where $u_{1}^{N}=\left(u_{1}, \ldots, u_{N}\right)$ are the input bits and $G_{N}$ is the generator matrix. Note that $G_{N}$ is constructed as

$$
G_{N}=B_{N} F^{\otimes n}
$$

where $B_{N}$ is the bit-reversal permutation matrix, $\otimes$ represents the Kronecker power, and $F$ is the kernel matrix which is defined as

$$
F \triangleq\left[\begin{array}{ll}
1 & 0 \\
1 & 1
\end{array}\right]
$$

The channel $W_{N}\left(y_{1}^{N} \mid u_{1}^{N}\right)$ can be split into a series of bitchannels $W_{N}^{(i)}: \mathscr{X} \longrightarrow \mathscr{Y}^{N} \times \mathscr{X}^{i-1}, 1 \leq i \leq N$, each channel $W_{N}^{(i)}$ with single input bit $u_{i}(1 \leq i \leq N)$, and its channel transition probabilities are given by

$$
W_{N}^{(i)}\left(y_{1}^{N}, u_{1}^{i-1} \mid u_{i}\right) \triangleq \sum_{u_{i+1}^{N} \in \mathcal{X}^{N-i}} \frac{1}{2^{N-1}} W_{N}\left(y_{1}^{N} \mid u_{1}^{N}\right)
$$

As $N$ tends to infinity, the capacity of the bit-channels $W_{N}^{(i)}$ will tend to 0 or 1 . In other words, this set of bitchannels will be polarized into noiseless and noisy channels. This phenomenon is called channel polarization. The idea is to transmit the information bits through the noiseless channels and transmit fixed bit known to both the transmitter and the receiver over the noisy channels. The codes constructed based on the idea of channel polarization are termed polar codes.

2.2. Construction of Polar Codes. The construction of polar codes can be briefly described as choosing bit-channels with low error probabilities to transmit information bits. Most of the studies quantize the error probabilities of the bitchannels to construct a polar code. Tal and Vardy introduce the concepts of the degraded and upgraded channels with finite output alphabet sizes to approximate the original channel [23]. Symbol-merging operations are employed after each convolution to obtain the degraded and upgraded channels. Denote by $Q$ and $Q^{\prime}$ the degraded and upgraded channels, respectively. Letting $P_{e}(W)$ be the error probability of channel $W$, the error probabilities of the original, degraded, and 
upgraded channels have the following relationship:

$$
P_{e}(Q) \geq P_{e}(W) \geq P_{e}\left(Q^{\prime}\right) .
$$

The upper and lower bounds on the error probabilities of the bit-channels are obtained through the evaluation of the degraded and upgraded channels.

2.3. Successive Cancellation Decoding Algorithm. The successive cancellation (SC) decoding algorithm decodes input bits sequently from $u_{1}$ to $u_{N}[1]$. Define the likelihood ratio (LR) as

$$
L_{N}^{(i)}\left(y_{1}^{N}, \widehat{u}_{1}^{i-1}\right) \triangleq \frac{W_{N}^{(i)}\left(y_{1}^{N}, \widehat{u}_{1}^{i-1} \mid 0\right)}{W_{N}^{(i)}\left(y_{1}^{N}, \widehat{u}_{1}^{i-1} \mid 1\right)},
$$

The $i$ th decision element $\widehat{u}_{i}$ can be represented as

$$
\widehat{u}_{i} \triangleq \begin{cases}u_{i}, & i \in \mathscr{A}^{c}, \\ 0, & i \in \mathscr{A}, L_{N}^{(i)}\left(y_{1}^{N}, \widehat{u}_{1}^{i-1}\right) \geq 1, \\ 1, & i \in \mathscr{A}, L_{N}^{(i)}\left(y_{1}^{N}, \widehat{u}_{1}^{i-1}\right)<1 .\end{cases}
$$
lows:

The likelihood ratios can be calculated reclusively as fol-

$$
\begin{aligned}
& L_{N}^{(2 i-1)}\left(y_{1}^{N}, \widehat{u}_{1}^{2 i-2}\right)=\frac{L_{N / 2}^{(i)}\left(y_{1}^{N / 2}, \widehat{u}_{1, o}^{2 i-2} \oplus \widehat{u}_{1, e}^{2 i-2}\right) L_{N / 2}^{(i)}\left(y_{N / 2+1}^{N}, \widehat{u}_{1, e}^{2 i-2}\right)+1}{L_{N / 2}^{(i)}\left(y_{1}^{N / 2}, \widehat{u}_{1, o}^{2 i-2} \oplus \widehat{u}_{1, e}^{2 i-2}\right)+L_{N / 2}^{(i)}\left(y_{N / 2+1}^{N}, \widehat{u}_{1, e}^{2 i-2}\right)}, \\
& L_{N}^{(2 i)}\left(y_{1}^{N}, \widehat{u}_{1}^{2 i-1}\right)= {\left[L_{N / 2}^{(i)}\left(y_{1}^{N / 2}, u \wedge_{1, o}^{2 i-2} \oplus u \wedge_{1, e}^{2 i-2}\right)\right]^{1-2 u \wedge_{2 i-1}} } \\
& \times L_{N / 2}^{(i)}\left(y_{N / 2+1}^{N}, \widehat{u}_{1, e}^{2 i-2}\right) .
\end{aligned}
$$

\section{Proposed Coding Scheme}

3.1. Structure of Proposed Coding Scheme. The Internet of Things has to support a large number of users. Data needs to be transmitted from multiple sources to single or multiple destinations. Each source has its own characteristics, such as data amount and reliability requirements. To meet these requirements, channel codes of different lengths and code rates are adopted. In the receiving end, multiple decoders need to be configured. Distributed coding is to encode data from multiple sources independently, and then, decode all coded sources jointly with a single decoder using the correlation between sources. Besides decreasing the number of decoders, the distributed codes can increase the overall code length, so that the distributed codes have the potential to improve the overall error correction performances. In this section, a distributed coding scheme of polar codes is proposed. The structure of our proposed coding scheme is given in Figure 1.

In this scheme, there is an encoder on each source; the original data are encoded and transmitted to the destination. To create correlation between the coded bits of various sources, there is a reconstructor in front of the decoder. As the output of reconstructor can be decoded using a single decoder, then the data from different sources can be considered as the information bits of a single polar code with longer code length than the subcodes on source.

3.2. Design of Reconstructor. Following the definition of distributed codes, by using the reconstructor to create correlation between the code bits of various sources, the original data on each source can be decoded using a common single decoder, then the proposed structures can be considered as a distributed code.

Let $G_{1}$ and $G_{2}$ denote the generator matrix of polar codes with code length $N_{1}=2^{n_{1}}$ and $N_{2}=2^{n_{2}}$, repectively. Assume $N_{1}>N_{2}$, we have

$$
G_{1}=G_{2} \otimes F^{\left(n_{1}-n_{2}\right)}
$$

Consider two polar codes with the same code lengths $N_{1}=2^{n_{1}}$. The two polar codes can be combined to a single polar code with $N_{\text {combined }}=2^{n_{1}+1}$. Let $G_{1}$ and $G_{2}$ denote the generator matrix of the two codes, the generating matrix of the combined code is

$$
G_{\text {combined }}=\left[\begin{array}{cc}
G_{1} & 0 \\
G_{2} & G_{2}
\end{array}\right] \text {. }
$$

Concerning our proposed scheme, the matrices of the subcodes are represented by $G_{1}, G_{2}, \cdots, G_{I}$, with the code lengths $N_{1}, N_{2}, \cdots, N_{I}$. For decoder, the collected code bits are considered as the codeword of a overall code with generating matrix $G_{\text {overall }}$ and code length $N_{\text {overall }}$. It is worth noting that $\sum_{i=1}^{I} N_{i}<N_{\text {overall }}$.

The encoding structure of the proposed scheme is illustrated in Figure 2. There are some subcodes with generating matrices $G_{j}^{0}$; the input bits of which are all zeros. Actually, the subcodes with all zero input bits are not transmitted and are only considered in the reconstructor, to make sure that the code length of the overall code is the power of two.

For simplicity, assuming that all the subcodes are with the same code length. As there are $I$ source subcodes and $J$ all-zero subcodes, the generator matrix of reconstructor is with code length $N_{R}=2^{n^{\prime}}=I+J$. The reconstructor is designed as a block-based code. And the code bits of each subcode are considered as an input block to the reconstructor. The generator matrix $G_{R}$ of the reconstructor has the same structure of a polar code, which can be represented as

$$
G_{R}=F^{\otimes \log _{2}(I+J)}=F^{\otimes n^{\prime}} .
$$

Note that the reconstructor is not an encoder of a polar code, as it is designed to encode the blocks.

Assume that all the subcodes on sources are with code length $N_{i}=2^{n}$, and the code length of reconstructor is $N_{R}=$ $2^{n^{\prime}}=I+J$, then the output bits can be generated with code length $N_{\text {overall }}=2^{n+n^{\prime}}$. And the generator matrix of the overall 


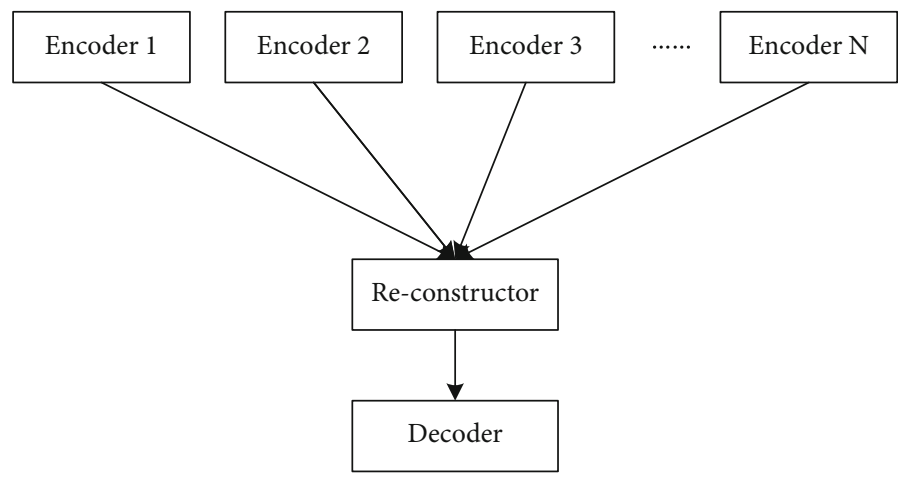

FIGURE 1: Structure of the proposed coding scheme.

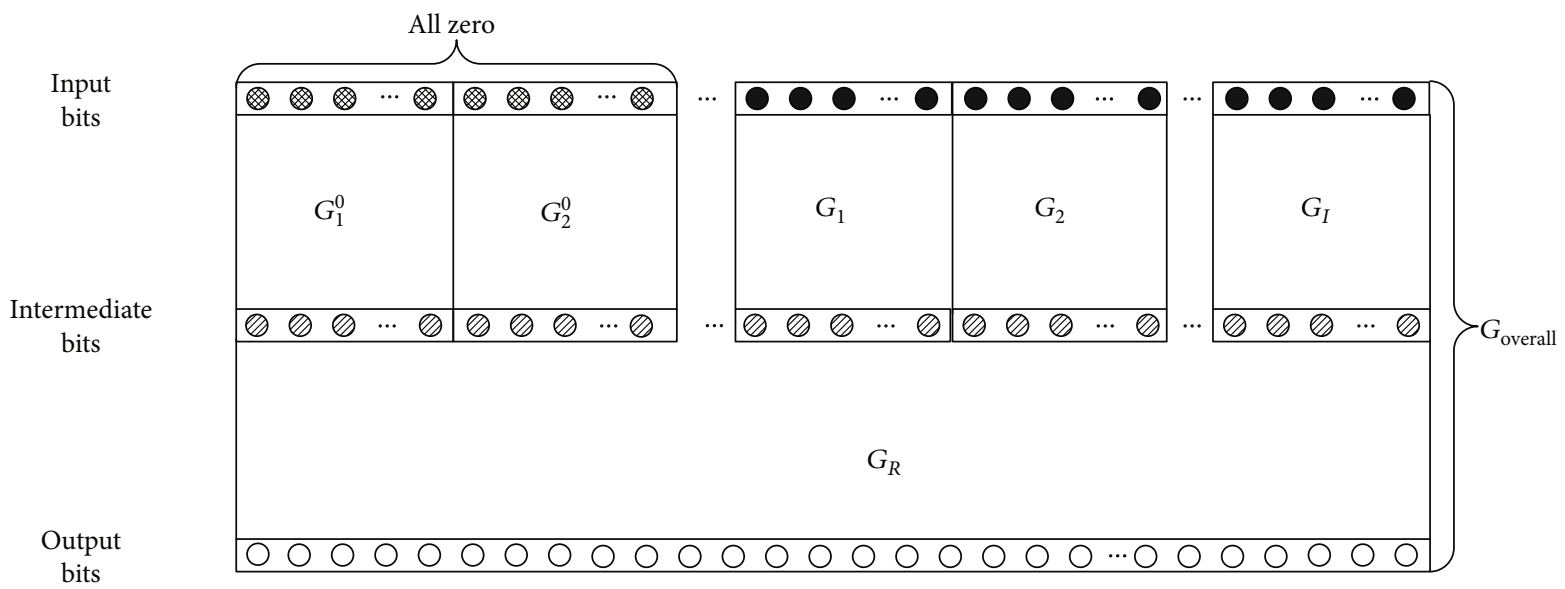

FIGURE 2: Encoding process of the proposed coding scheme.

distributed code can be computed using the following equation.

$$
G_{\text {overall }}=F^{\otimes n^{\prime}} \otimes G_{N_{i}}
$$

As can be found in Figure 2, the code bits of subcodes are termed intermediate bits, and the intermediate bits would be recoded into output bits. There is a significant difference between the traditional polar codes and the recode in our proposed scheme. For traditional polar codes, the information bits are without any errors. However, the proposed coding scheme has to deal with intermediate bits with noise. The input blocks of the reconstructor come from two ways, the all-zero subcodes and the subcodes on sources. The all-zero subcodes are virtual sequences which are not transmitted, so that these intermediate bits are error free. For the subcodes on sources, as the reconstructor is set in front of the decoder, the intermediate bits are transmitted through channels, so that the related intermediate bits are affected by the noise of the channels.

Consider a distributed polar code with two all-zero subcodes and two subcodes on sources. The generator matrices of the subcodes are the submatrices of the overall generator matrix. Let $G_{1}^{0}, G_{2}^{0}, G_{1}$, and $G_{2}$ denote the generator matrices of the subcodes, respectively. Note that these submatrices are actually same to each other. The generator matrix of recon- structor is given by

$$
G_{R}=\left[\begin{array}{llll}
1 & 0 & 0 & 0 \\
1 & 1 & 0 & 0 \\
1 & 0 & 1 & 0 \\
1 & 1 & 1 & 1
\end{array}\right] .
$$

Then, the overall generator matrix $G_{\text {overall }}$ is given by

$$
G_{\text {overall }}=\left[\begin{array}{cccc}
G_{1}^{0} & 0 & 0 & 0 \\
G_{2}^{0} & G_{2}^{0} & 0 & 0 \\
G_{1} & 0 & G_{1} & 0 \\
G_{2} & G_{2} & G_{2} & G_{2}
\end{array}\right] \text {. }
$$

\section{Discussion on the Existing Issues of Proposed Scheme}

There are two main issues that need to be elaborated while adopting the proposed coding scheme. One is how the proposed coding scheme can provide UEP property. The other is how to reconstruct the collected code bits of the subcodes and generate the output bits on the condition that collected 
code bits are affected by the noises. In this section, these two issues will be analyzed and discussed.

4.1. UEP Property of the Proposed Coding Scheme. Firstly, the UEP property of the proposed coding scheme is concerned. As the proposed distributed coding scheme mainly follows the construction of polar codes, the BLER performances of the proposed coding scheme also follows the property of polar codes.

As is known to us, for a given code with fixed code length, the BLER performances of a given code with well-designed parameters are mainly determined by their own code rates. A higher code rate would lead to a larger BLER. This means the BLER performances of the subcodes are mainly determined by their own code rates. Then, Proposition 1 can be given.

Proposition 1. For a given proposed distributed code, the subcodes with lower code rates would have better BLER performances than the other subcodes with higher code rates.

There is another factor that would be affected on the BLER performances of subcodes besides code rates. We name this factor as the error accumulation phenomenon. Let $\varepsilon$ denote the block error event which is defined as $\varepsilon \triangleq\left\{\widehat{u}_{1}^{N} \neq\right.$ $\left.u_{1}^{N}\right\}$, the BLER of a given polar code can be calculated as [24].

$$
P(\varepsilon)=1-\prod_{i \in \mathscr{A}}\left(1-P_{e}\left(W_{N}^{(i)}\right)\right),
$$

where $P_{e}\left(W_{N}^{(i)}\right)$ is the error probability of the $i$ th bit-channel and $A$ is the information set.

Divide the information set $A$ into a series subsets $s_{1}, s_{2}, \cdots, s_{r}$ in accending order of their indices. That is, for subsets $s_{p}$ and $s_{q}$, if $p<q$, all the information bits in $s_{p}$ have lower bit indices than that in $s_{q}$. Let $P\left(\varepsilon_{s_{p}}^{U}\right)$ and $P\left(\varepsilon_{s_{q}}^{U}\right)$ denote the upper bounds on the BLERs of $s_{p}$ and $s_{q}$. Following eqn. (26) in [16], we have

$$
P\left(\varepsilon_{s_{p}}^{U}\right)<P\left(\varepsilon_{s_{q}}^{U}\right)
$$

Similarly, let $P\left(\varepsilon_{s_{p}}^{L}\right)$ and $P\left(\varepsilon_{s_{q}}^{L}\right)$ represent the lower bounds on the BLERs of $s_{p}$ and $s_{q}$. We have

$$
P\left(\varepsilon_{s_{p}}^{L}\right)<P\left(\varepsilon_{s_{q}}^{L}\right) .
$$

Then, the following proposition can be given.

Proposition 2. For a given proposed distributed code, the subcodes are indexed according to their positions in the reencoding process as in Figure 2. If the information sets of the subcodes are selected jointly as a single polar code, and the affection proposed in Proposition 1 is not considered, then the subcodes with smaller indices have better BLER performances than the ones with larger indices.

The third factor which would affect the BLER performances of subcodes is discussed below. As for the decoder in the proposed coding scheme, the output bits are considered as the code bits of a single polar code, then the information set can be selected jointly as determining whether $i \in \mathscr{A}_{\theta}$, where $\mathscr{A}_{\theta} \triangleq\left\{i \in\{1, \cdots, N\}: P_{e}\left(W_{N}^{(i)}\right)<\theta\right\}$, and $\theta \in[0,1]$ is the threshold. The information bits selected this way are more likely to be located in the back part of the code. Assume that the subcodes are indexed according to their positions. That is, the subcodes in the front have smaller indices. For two subcodes $C_{p}$ and $C_{q}$, if $p<q$, we can usually have $\left|A_{p}\right|$ $<\left|A_{q}\right|$. Note that this is determined by the code length, code rate of the single code and code length, code indices of the subcodes. On the other hand, assume the code rate of the subcodes $C_{p}$ and $C_{q}$ are the same, which means $\left|A_{p}\right|=\left|A_{q}\right|$, then we have $\theta_{p}>\theta_{q}$, which means the average information bit-channel quality of $C_{p}$ is worse than that of $C_{q}$.

Proposition 3. For a given proposed distributed code, if the subcodes have the same code rate, regardless of the affection stated in Proposition 2, the subcodes with larger indices would have better BLER performances than the ones with smaller indices.

4.2. Combination of Coded Bits. In this subsection, the issue of how to reconstruct the code bits and generating the output bits will be addressed. Consider a distributed polar code with $I$ source subcodes and $J$ all-zero subcodes as mentioned above. Assume that all the subcodes are with code length $N_{i}=2^{n}$ and that $I+J=2^{n^{\prime}}$. Let $U_{i}=\left\{u_{i, 1}, u_{i, 2}, \cdots, u_{i, 2^{n}}\right\}$ denote the block of input bits of subcode $C_{i}$, then the coded bits of this subcode is denoted by $X_{i}=\left\{x_{i, 1}, x_{i, 2}, \cdots, x_{i, 2^{n}}\right\}$. The corresponding collected bits after being transmitted through the channels are denoted by $Z_{i}=\left\{z_{i, 1}, z_{i, 2}, \cdots, z_{i, 2^{n}}\right\}$ . Define the intermediate bits as $\mathscr{Z}=\left\{Z_{1}, Z_{2}, \cdots, Z_{2^{n^{\prime}}}\right\}$. It is worth noting that there are $J$ block of all-zero subcodes which are not practical transmitted through the channels. The intermediate bits of these subcodes are also all-zero blocks. After reencoding the intermediate bits, the output bits are denoted by $\mathcal{Y}=\left\{Y_{1}, Y_{2}, \ldots, Y_{2^{n^{\prime}}}\right\}=\left\{y_{1}, y_{2}, \ldots, y_{2^{n+n^{\prime}}}\right\}$.

In the reconstructing process, the intermediate bits are reencoded. As the intermediate bits of source subcodes are affected by noises, the reencoding process of distributed polar code is entirely different from the encoding process of traditional polar codes. In this paper, we deal with the bits with noises from the likelihood ratio (LR) perspective. The LR of an output bit which is combined using two intermediate bits can be computed using the following theorem.

Theorem 4. Assuming that an output bit $y_{i}$ is combined using intermediate bits $z_{j_{1}}$ and $z_{j_{2}}$, the process of which is defined as $y_{i}=z_{j_{1}} \boxplus z_{j_{2}}$. The LR of $y_{i}$ is given by

$$
L\left(y_{i}\right)=\frac{L\left(z_{j_{1}}\right) L\left(z_{j_{2}}\right)+1}{L\left(z_{j_{1}}\right)+L\left(z_{j_{2}}\right)} .
$$




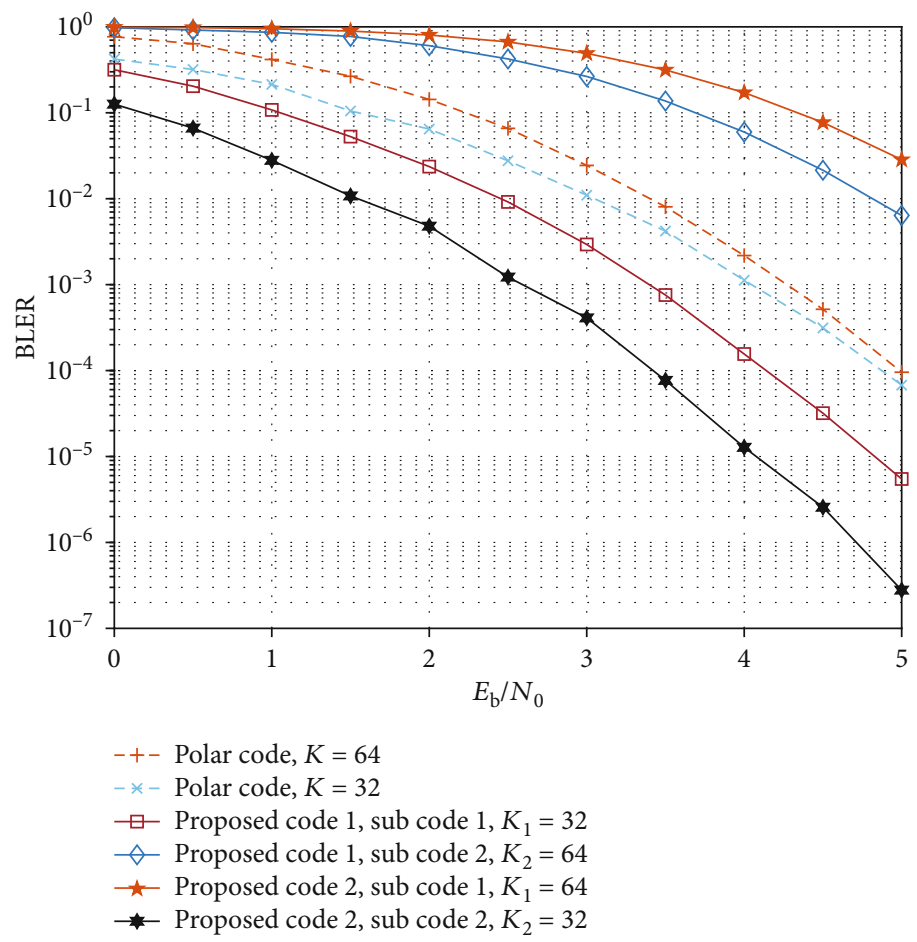

FIGURE 3: BLER performances of the proposed coding scheme with two subcodes. The number of information bits in the two subcodes are 32 and 64.

Proof. The LR of $y_{i}$ can be expressed by

$$
\begin{aligned}
L\left(y_{i}\right)= & \frac{\operatorname{Pr}\left(y_{i} \mid 0\right)}{\operatorname{Pr}\left(y_{i} \mid 1\right)}=\frac{\operatorname{Pr}\left(z_{j_{1}} \boxplus z_{j_{2}} \mid x_{j_{1}} \boxplus x_{j_{2}}=0\right)}{\operatorname{Pr}\left(z_{j_{1}} \boxplus z_{j_{2}} \mid x_{j_{1}} \boxplus x_{j_{2}}=1\right)} \\
= & \frac{\operatorname{Pr}\left(z_{j_{1}} \boxplus z_{j_{2}} \mid x_{j_{1}}=0, x_{j_{2}}=0\right)+\operatorname{Pr}\left(z_{j_{1}} \boxplus z_{j_{2}} \mid x_{j_{1}}=1, x_{j_{2}}=1\right)}{\operatorname{Pr}\left(z_{j_{1}} \boxplus z_{j_{2}} \mid x_{j_{1}}=1, x_{j_{2}}=0\right)+\operatorname{Pr}\left(z_{j_{1}} \boxplus z_{j_{2}} \mid x_{j_{1}}=0, x_{j_{2}}=1\right)},
\end{aligned}
$$

As the intermediate bits $z_{j_{1}}$ and $z_{j_{2}}$ are independent, then (21) can be rewritten as

$$
\begin{aligned}
L\left(y_{i}\right) & =\frac{P\left(z_{j_{1}} \mid x_{j_{1}}=0\right) P\left(z_{j_{2}} \mid x_{j_{2}}=0\right)+P\left(z_{j_{1}} \mid x_{j_{1}}=1\right) P\left(z_{j_{2}} \mid x_{j_{2}}=1\right)}{P\left(z_{j_{1}} \mid x_{j_{1}}=1\right) P\left(z_{j_{2}} \mid x_{j_{2}}=0\right)+P\left(z_{j_{1}} \mid x_{j_{1}}=0\right) P\left(z_{j_{2}} \mid x_{j_{2}}=1\right)} \\
& =\frac{\left(P\left(z_{j_{1}} \mid x_{j_{1}}=0\right) / P\left(z_{j_{1}} \mid x_{j_{1}}=1\right)\right)\left(P\left(z_{j_{2}} \mid x_{j_{2}}=0\right) / P\left(z_{j_{2}} \mid x_{j_{2}}=1\right)\right)+1}{P\left(z_{j_{1}} \mid x_{j_{1}}=0\right) / P\left(z_{j_{1}} \mid x_{j_{1}}=1\right)+P\left(z_{j_{2}} \mid x_{j_{2}}=0\right) / P\left(z_{j_{2}} \mid x_{j_{2}}=1\right)} \\
& =\frac{L\left(z_{j_{1}}\right) L\left(z_{j_{2}}\right)+1}{L\left(z_{j_{1}}\right)+L\left(z_{j_{2}}\right)} .
\end{aligned}
$$

Repeating the combining process of intermediate bits as shown in (20), all the output bits can be generated.

\section{Simulation Results}

In this section, we evaluate the performance of the proposed coding scheme via simulations. As the IoT services usually have short-packet characteristics, all the $E_{\mathrm{b}} / N_{0}$ in the following figures is the $E_{\mathrm{b}} / N_{0}$ of the system. The code lengths of subcodes are set to 128 . For simplicity, we first consider the proposed scheme with 2 subcodes. As the number of sources is a power of two, there is no need for all-zero blocks. The number of information bits for the two subcodes are 32 and 64 , respectively. Note that the combination order of the two subcodes has influences on the performance. The BLER performances of the two subcodes under both combination orders have been plotted. The BLER curves of $(128,64)$ polar code and $(128,32)$ polar code have been plotted as comparison. In Figure 3, for proposed code 1, the number of information bits of the subcodes are $K_{1}=32$ and $K_{2}=64$. It can be seen that subcode 1 can provide better BLER performance than its pair. Compared with the traditional polar codes with $K=32$ and $K=64$ of the same code length, subcode 1 in proposed code 1 can provide better BLER performance at the price of decreasing the BLER performance on its pair subcode 2. For proposed code 2 , the subcodes are with $K_{1}=64$ and $K_{2}=32$. Subcode 2 of proposed code 2 has better BLER performance than subcode 1 of proposed code 1 and the traditional polar code with $K=32$. Meanwhile, subcode 1 of proposed code 2 has the worst BLER performance. It can be seen that the proposed coding scheme has shown evident UEP property. One can easily find that in both proposed codes 1 and 2, the subcodes with lower code rates can provide better 


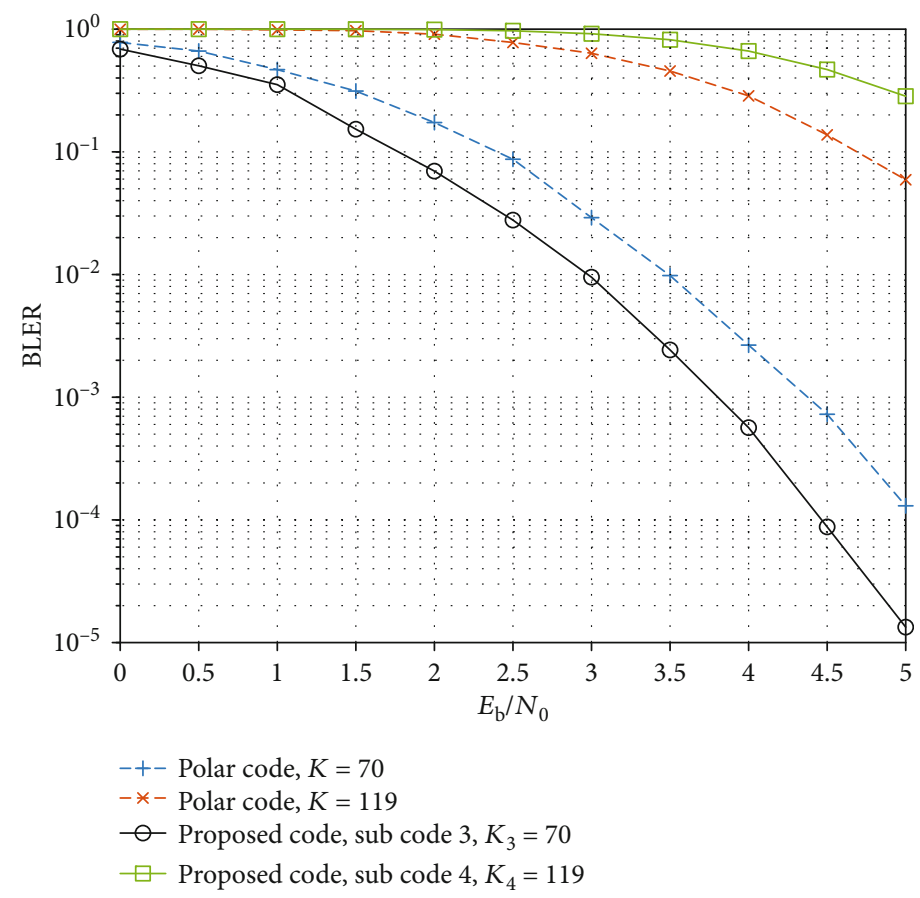

FIgURE 4: BLER performances of the proposed coding scheme with four subcodes. The first two are virtual subcodes; the number of information bits in the third and fourth subcodes are 70 and 119.

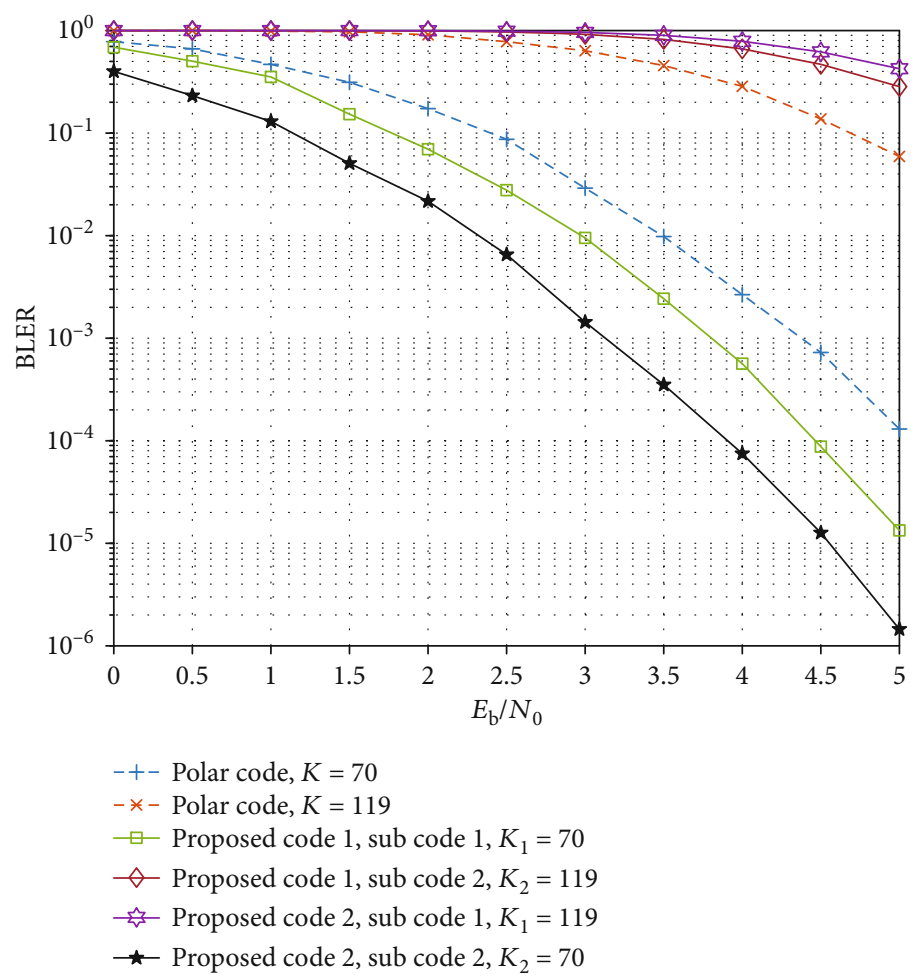

Figure 5: BLER performances of the proposed coding scheme with two subcodes. The number of information bits in the two subcodes are 70 and 119 . 


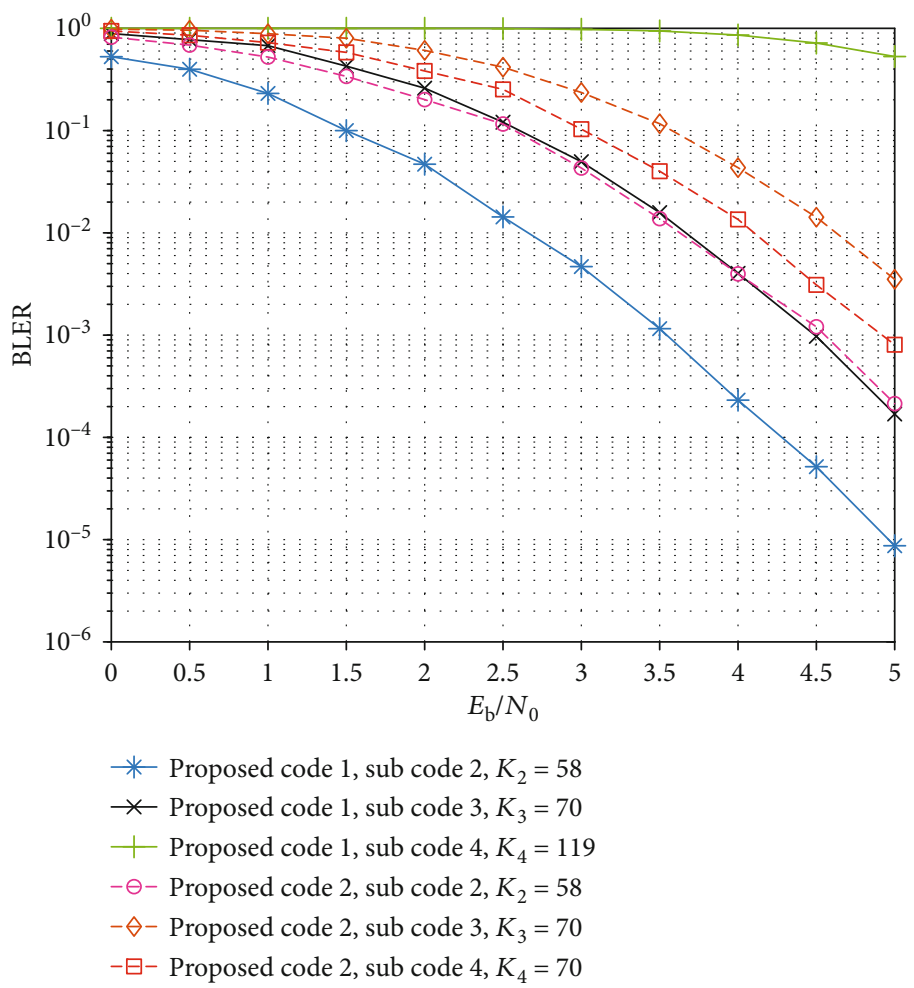

FIGURE 6: BLER performances of the proposed coding scheme with four subcodes. The first one is virtual subcode; the number of information bits in the second, third, and fourth subcodes in proposed code 1 and proposed code 2 is 58, 70, 119 and 58, 70, 70, respectively.

BLER performances than their pairs. This is consistent with the phenomenon shown in Proposition 1. Comparing the subcodes with 32 information bits in both proposed codes 1 and 2, one can find that the subcodes with larger subcode indices have the better BLER performances, which means that although the phenomenons proposed in Proposition 2 and Proposition 3 both have effects on the proposed codes, and the affection of Proposition 3 is more significant than that of the Proposition 2.

In the proposed coding scheme, the number of subcodes is a power of 2 . However, in many scenarios, the number of sources does not meet this requirement. To solve this problem, all-zero subcodes are introduced in the proposed scheme. For simplicity, we consider a proposed code with 4 subcodes, in which the first 2 subcodes are all-zero subcodes, and for subcodes 3 and 4, the number of information bits are $K_{3}=70$ and $K_{4}=119$. As shown in Figure 4, compared with the traditional polar codes with the same code length and same size of information sets, subcode 3 can provide better BLER performance at the price of decreasing the BLER performance of subcode 4 .

The first two subcodes in Figure 4 are all-zero blocks. In Figure 5, we evaluate the performance of the proposed coding scheme without these two all-zero blocks. That is, only two subcodes are considered. The number of information bits is the same as in Figure 4. Both combination orders are considered. In proposed codes 1 and 2 , the number of information bits is $K_{1}=70, K_{2}=119$, and $K_{1}$ $=119, K_{2}=70$, respectively. Compared with the tradi- tional polar codes with $K=70$ and $K=119$, the subcodes of proposed codes with less information bits can provide better BLER performances than traditional ones. Meanwhile, the subcodes with more information bits would lead to worse BLER performances. Moreover, the BLER performance of the subcodes in proposed code 1 is almost the same as that of subcode 3 and subcode 4 shown in Figure 4. This illustrates that the all-zero subcodes cannot improve the BLER performance of the distributed code. The function of all-zero subcodes is to make up the number of subcodes to an integer power of 2 .

As all-zero subcodes cannot provide obvious benefits on BLER performances of the proposed distributed coding scheme, and the larger number of all-zero subcodes would lead to higher reconstructing and decoding complexities, all-zero subcodes are only used to complement the number of subcodes into a power of 2. The BLER performances of the proposed codes with 1 all-zero subcode and 3 source subcodes are given in Figure 6. It can be found that in the proposed code 1 , the subcode with lower code rate can provide better BLER performance than its pair, and the UEP performances among the subcodes are obvious. By arbitrarily changing the code rate of subcode 4 , the BLER performance of subcode 4 in proposed code 2 can be dramatically improved, at the cost of the BLER performance of subcode 3 getting worse, and much worse than that of subcode 3 in proposed code 1 . As in proposed code 2 , subcode 4 has better BLER performance than subcode 3 , which is consistent with Proposition 3. 


\section{Conclusion}

In this paper, a distributed coding scheme based on polar codes which can provide UEP property is proposed to support the varied data transmission in the IoT scenarios. By focusing on the challenge that the distributed codes are not workable over noisy channels, we put forward a novel method to combine the noisy bits under our proposed coding structures, by giving the mathematical model and deriving the recursive expressions of the combination process of the noisy bits from the LR prospective. The proposed distributed polar codes can be adopted in the IoT scenarios to transmit the data of various sources using a single common decoder. And based on the intuitive sense of the error propagation in the SC decoding process, the proposed codes can provide obvious UEP properties between the data of different sources. The simulation results proved that the data of the multiple sources of IoT can be decoded successfully using a single common decoder, and the various reliability requirements of the data from different sources can be satisfied through the obvious UEP property.

\section{Data Availability}

The data used to support the findings of this study are available from the corresponding author upon request.

\section{Conflicts of Interest}

The authors declare that they have no conflicts of interest.

\section{Acknowledgments}

This work was supported by the Beijing Natural Science Foundation under grant L182032, the National Natural Science Foundation of China (No. 61601147), the Development Foundation of CECT54 (Grant No. XX17641C008), and the Shaanxi S\&T under grant 2021KW-07.

\section{References}

[1] E. Arikan, "Channel polarization: a method for constructing capacity-achieving codes for symmetric binary-input memoryless channels," IEEE Transactions on Information Theory, vol. 55, no. 7, pp. 3051-3073, 2009.

[2] Y. P. Eric Wang, X. Lin, A. Adhikary et al., "A primer on 3GPP narrowband Internet of Things (NB-IoT)," IEEE Communications Magazine, vol. 55, no. 3, 2016.

[3] M. Lauridsen, B. Vejlgaard, I. Z. Kovacs, H. Nguyen, and P. Mogensen, "Interference measurements in the European $868 \mathrm{MHz}$ ISM band with focus on LoRa and SigFox," in In Wireless Communications \& Networking Conference, 2017.

[4] S. H. Weidang Lu, X. Liu, C. He, and Y. Gong, "Incentive mechanism based cooperative spectrum sharing for OFDM cognitive IoT network," IEEE Transactions on Network Science and Engineering, vol. 7, no. 2, pp. 662-672, 2020.

[5] W. U. Khan, J. Liu, F. Jameel, V. Sharma, R. Jantti, and Z. Han, "Spectral efficiency optimization for next generation NOMAenabled IoT networks," IEEE Transactions on Vehicular Technology, vol. 69, no. 12, pp. 15284-15297, 2020.
[6] X. Liu, X. Zhai, W. Lu, and C. Wu, "QoS-guarantee resource allocation for multibeam satellite industrial Internet of Things with NOMA," IEEE Transactions on Industrial Informatics, vol. 17, no. 3, pp. 2052-2061, 2021.

[7] X. Liu and X. Zhang, "NOMA-based resource allocation for cluster-based cognitive industrial Internet of Things," IEEE Transactions on Industrial Informatics, vol. 16, no. 8, pp. 5379-5388, 2020.

[8] M. Dong, G. Lan, M. Hassan, W. Hu, and S. K. Das, "Sensing, computing, and communications for energy harvesting IoTs: a survey," IEEE Communications Surveys Tutorials, vol. 22, no. 2, pp. 1222-1250, 2020.

[9] A. O. Ercan, M. O. Sunay, and I. F. Akyildiz, "RF energy harvesting and transfer for spectrum sharing cellular IoT communications in 5G systems," IEEE Transactions on Mobile Computing, vol. 17, no. 7, pp. 1680-1694, 2018.

[10] X. Liu and X. Zhang, "Rate and energy efficiency improvements for 5G-based IoT with simultaneous transfer," IEEE Internet of Things Journal, vol. 6, no. 4, pp. 5971-5980, 2019.

[11] G. Caire and G. Lechner, "Turbo codes with unequal error protection,” Electronics Letters, vol. 32, no. 7, pp. 629-631, 1996.

[12] V. Kumar and O. Milenkovic, "On unequal error protection LDPC codes based on plotkin-type constructions," IEEE Transactions on Communications, vol. 54, no. 6, pp. $994-$ 1005, 2006.

[13] N. Rahnavard, H. Pishro-Nik, and F. Fekri, "Unequal error protection using partially regular LDPC codes," IEEE Transactions on Communications, vol. 55, no. 3, pp. 387-391, 2007.

[14] N. Rahnavard, B. N. Vellambi, and F. Fekri, "Rateless codes with unequal error protection property," IEEE Transactions on Information Theory, vol. 53, no. 4, pp. 1521-1532, 2007.

[15] D. Sejdinovic, D. Vukobratovic, A. Doufexi, V. Senk, and R. J. Piechocki, "Expanding window fountain codes for unequal error protection," IEEE Transactions on Communications, vol. 57, no. 9, pp. 2510-2516, 2009.

[16] C. Cui, W. Xiang, Z. Wang, and Q. Guo, "Polar codes with the unequal error protection property," Computer Communications, vol. 123, pp. 116-125, 2018.

[17] D. Slepian and J. K. Wolf, "Noiseless coding of correlated information sources," IEEE Transactions on Information Theory, vol. 19, no. 4, pp. 471-480, 1973.

[18] N. Deligiannis, J. Barbarien, M. Jacobs, A. Munteanu, A. Skodras, and P. Schelkens, "Side-information-dependent correlation channel estimation in hash-based distributed video coding," IEEE Transactions on Image Processing, vol. 21, no. 4, pp. 1934-1949, 2012.

[19] S. Wang, L. Cui, L. Stankovic, V. Stankovic, and S. Cheng, "Adaptive correlation estimation with particle filtering for distributed video coding," IEEE Transactions on Circuits \& Systems for Video Technology, vol. 22, no. 5, pp. 649-658, 2012.

[20] A. Talari and N. Rahnavard, "Distributed unequal error protection rateless codes over erasure channels: a two-source scenario," IEEE Transactions on Communications, vol. 60, no. 8, pp. 2084-2090, 2012.

[21] A. Talari and N. Rahnavard, "Distributed rateless codes with UEP property," in In Proc. IEEE International Symposium on Information Theory, pp. 2453-2457, Austin, TX, 2010.

[22] S. Wu, D. Li, Z. Wang, and Q. Guo, "Novel distributed UEP rateless coding scheme for data transmission in deep space networks," Science China, vol. 61, 2018. 
[23] I. Tal and A. Vardy, "How to construct polar codes," IEEE Transactions on Information Theory, vol. 59, no. 10, pp. 6562-6582, 2013.

[24] D. Wu, Y. Li, and Y. Sun, "Construction and block error rate analysis of polar codes over AWGN channel based on Gaussian approximation," IEEE Communications Letters, vol. 18, no. 7, pp. 1099-1102, 2014. 